\title{
FÍSTULA VÉSICOUTERINA, UNA RARA COMPLICACIÓN DEL PARTO POR CESÁREA
}

\author{
N. MEDINA RAMOS*, J.F. CEREZUELA REQUENA*, A. MARTÍN MARTÍNEZ*, \\ J.A. GARCÍA HERNÁNDEZ*, N. CHESA PONCE** \\ Unidad de Ginecología Urológica y Patología del Suelo Pélvico. *Servicio de Obstetricia y Ginecología. \\ Hospital Universitario Materno Infantil de Canarias. ${ }^{* *}$ Servicio de Urología. \\ Hospital Universitario Insular de Gran Canaria.
}

Actas Urol Esp. 27 (3): 244-247, 2003

\section{RESUMEN}

"FÍSTULA VÉSICOUTERINA, UNA RARA COMPLICACIÓN DEL PARTO POR CESÁREA"

Presentamos el caso de una fístula vésicouterina secundaria a una cesárea indicada por desproporción pélvico-cefálica.

Este tipo de fístulas se debe fundamentalmente a causas obstétricas, sobre todo a cesáreas en países desarrollados y a partos prolongados en países en vías de desarrollo.

La presentación clínica más común es la incontinencia urinaria, manifestándose como escapes urinarios continuos o intermitentes.

El tratamiento quirúrgico es generalmente la terapéutica de elección, aunque en casos de fístulas pequeñas el tratamiento conservador es factible.

La mejor prevención consiste en una correcta indicación de la cesárea así como una cuidadosa técnica quirúrgica.

PALABRAS CLAVE: Fístula vésicouterina. Etiología. Tratamiento quirúrgico.

\section{ABSTRACT}

"VESICOUTERINE FISTULA, A RARE COMPLICATION OF CAESAREAN SECTION BIRTH"

We present the case of a vesicouterine fistula secondary to a caesarean section indicated due to the disproportion the pelvis and the head of the baby.

This kind of fistula is due fundamentally to obstetric causes, especially to caesarean sections in developed countries and to prolonged labour in developing contries.

The commonest clinical presentation is urinary incontinence in the form of continuous or intermittent urinary leaks.

Surgical treatment is generally the therapy of choice, although, in the case of small fistulas, conservative treatment is feasible.

The best form of prevention is correct indication of caesareean section and careful surgical technique.

KEY WORDS: Vesicouterina fistula. Etiology. Surgical treatment. 
$\mathrm{L}$ a fístula vésicouterina constituye el 4\% de las fístulas urogenitales, ocupando el tercer lugar en frecuencia tras las urétero-vaginales y las vésicovaginales $^{1}$.

$\mathrm{Su}$ incidencia actual no es bien conocida ${ }^{2}$ debido probablemente a su baja prevalencia, aunque se sospecha su incremento en función del mayor número de partos quirúrgicos.

Tancer ${ }^{3}$ encuentra sólo 18 casos publicados entre 1908-1945, incrementándose la prevalencia a 74 casos entre 1946-1986.

Shing ${ }^{4}$ demuestra en su revisión bibliográfica un marcado aumento del número de fístulas vésicouterinas entre 1986-1997 con un total de 64 nuevos casos, de las que el $83 \%$ son secundarios a una cesárea.

\section{CASO CLÍNICO}

Paciente de 36 años, remitida a la Unidad de Ginecología Urológica del HUMIC por comenzar con escapes urinarios al mes de practicársele una cesárea por desproporción pélvico-fetal.

Como antecedentes personales, presenta intervención de fístula sacrococcígea y antecedentes obstétricos de un parto por cesárea en 1989 y un parto distócico mediante fórceps por expulsivo prolongado en 1994.

Tras tratamiento de una infección del tracto urinario con posterior urocultivo negativo, es valorada en nuestra Unidad, destacando en la anamnesis escapes continuos de orina clara que se desencadenan con la bipedestación y que aumentan con los esfuerzos, sin sintomatología de urgencia miccional acompañante.

En la exploración física general se aprecia abdomen blando y depresible sin puntos dolorosos, masas ni megalias, siendo el resto de la exploración por sistemas y aparatos normal. En cuanto a la exploración ginecológica presenta unos genitales externos y vagina de características normales, cérvix de multípara bien epitelizado, útero en anteversión, de tamaño, forma y consistencia normales, zonas anexiales libres de masas, no palpándose anejos.

La sensibilidad vulvar y los reflejos perineales (anal y bulbocavernoso) están conservados.

Tras realizar un test de incontinencia con azul de metileno, se objetiva a través del orificio cer- vical externo fluir líquido teñido de azul de forma espontánea, que aumenta con maniobras de Valsalva, no apreciándose escapes a través de uretra.

Confirmada la comunicación génitourinaria se solicita una cistografía en la que se comprueba, con vejiga en máxima repleción, un paso de contraste a través de un trayecto fistuloso a cavidad uterina (Fig. 1).

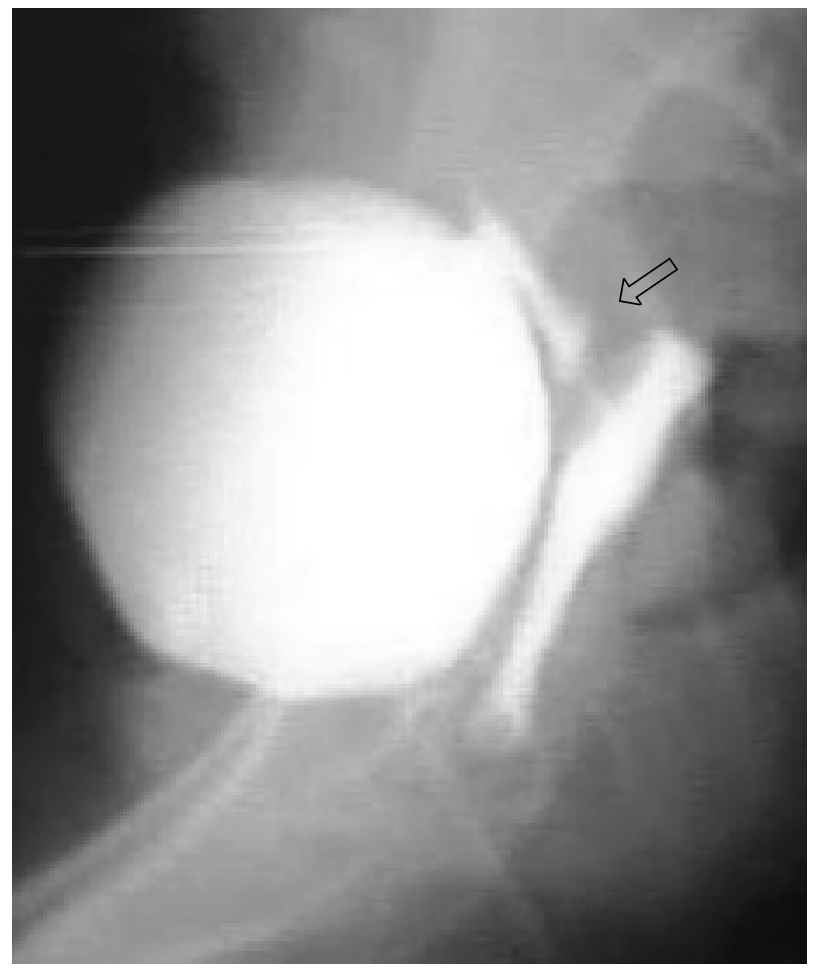

FIGURA 1

Una vez diagnosticada se remite la paciente al Servicio de Urología del Hospital Universitario Insular de Gran Canaria, donde se completa el estudio con la realización de una urografía que informa de imagen vesical normal con escape de contraste a nivel de cúpula (Figs. 2 y 3). En la uretrocistoscopia se objetiva orificio fistuloso en línea media de cara posterior y cúpula vesical. Tras conocer la localización y extensión del proceso fistuloso se le practica bajo anestesia general fistulorrafia vésicouterina por laparotomía infraumbilical; posteriormente el post-operatorio evoluciona de forma satisfactoria. 


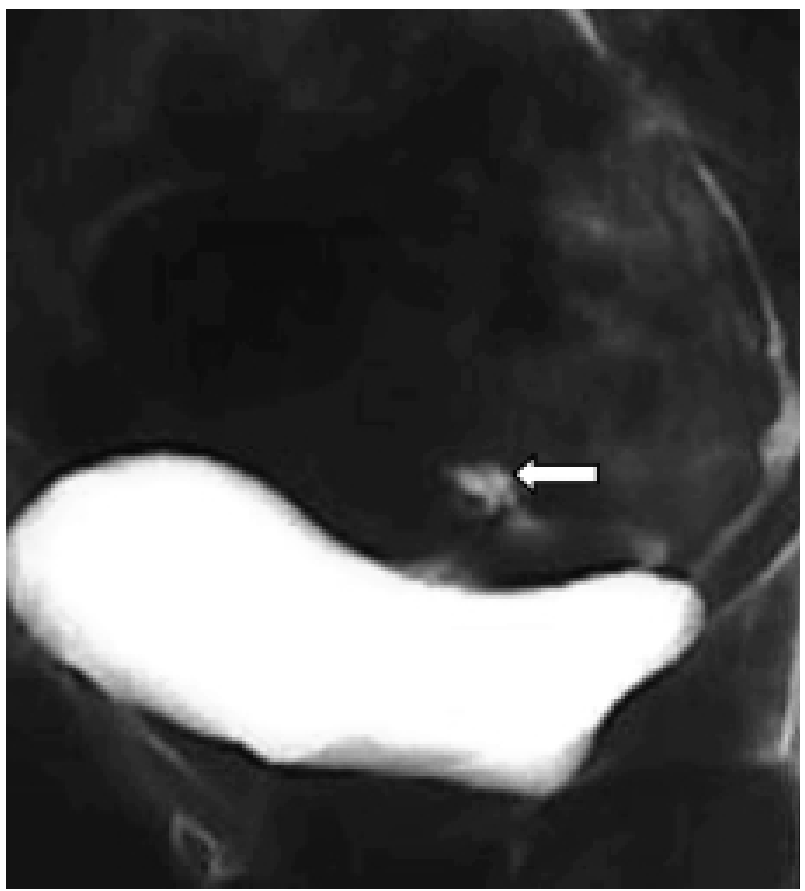

FIGURA 2

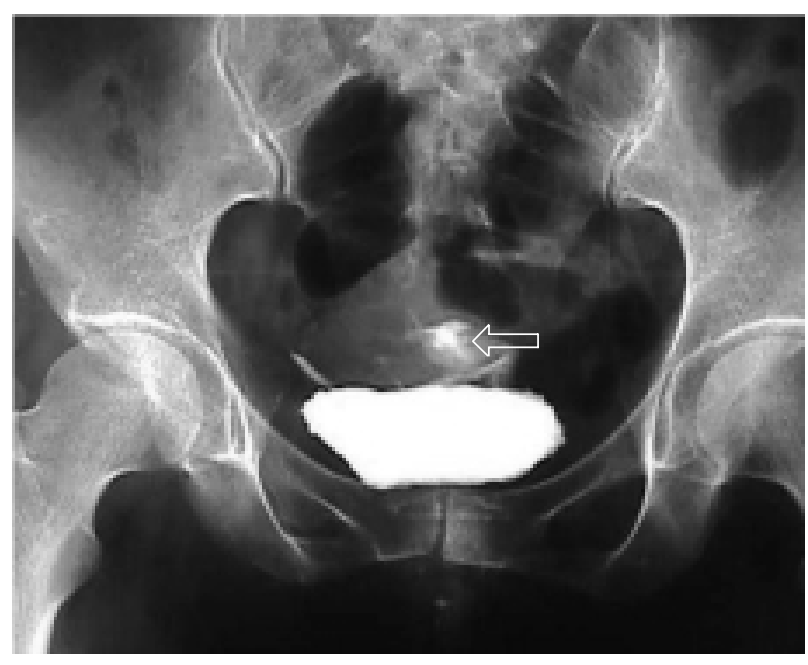

FIGURA 3

\section{DISCUSION}

La etiología más frecuente de la fístula vésicouterina, tanto en países subdesarrollados como desarrollados, es la obstétrica. En el primer caso se debe fundamentalmente a los partos muy prolongados, en los que la vejiga sufre un proceso de necrosis por presión entre la cabeza fetal y la sínfisis del pubis.

En los países desarrollados, a lo largo de la centuria pasada, se observa una inversión de la etiología, de tal manera que en la primera mitad del siglo el $61 \%$ de los casos de fístulas se debían a procedimientos obstétricos vaginales, siendo el porcentaje de partos por vía abdominal entre un 2 y un $5 \%$. Desde la segunda mitad de siglo hasta la actualidad las cesáreas se incrementan hasta un $25 \%$ o más, representando el $80 \%$ de las causas ${ }^{3}$.

Si bien el parto abdominal es la causa principal de fístulas vésicouterinas, éstas complican sólo el 0,04\% de las cesáreas ${ }^{5}$, cuyo mecanismo de producción principal es la necrosis de la pared vesical tras ser atrapada de forma inadvertida en la sutura de la histerorrafia.

El antecedente de cesárea previa aumenta el riesgo de fístula vésicouterina ${ }^{6,7}$, llegando a alcanzar hasta el doble ${ }^{2}$, debido a la mayor adherencia existente entre la cara posterior vesical y la cicatriz uterina. Otros casos más raros, con antecedente de cesárea previa, han sido descritos tras parto instrumental mediante fórceps y ventosa, tras parto eutócico e incluso tras extracción manual de placenta.

La perforación uterina, con alcance vesical tras legrado evacuador o tras colocación de DIU, es menos frecuente.

Otras causas descritas son la infiltración tumoral, la radioterapia a nivel de pelvis, infecciones pélvicas secundarias a tuberculosis o actinomicosis, siendo estas últimas prácticamente anecdóticas.

Algunas fístulas se desarrollan en el post-operatorio inmediato, indicando un traumatismo directo. En otras ocasiones se manifiesta tardíamente en el puerperio, reflejando probablemente el grado de afectación del trofismo local. La presentación clínica puede ser variable, siendo el síntoma más común la incontinencia urinaria, la cual puede presentarse como escapes urinarios continuos o intermitentes en función del tamaño y localización de la fístula ${ }^{8}$. En algunas ocasiones, por la presencia de mecanismo valvular a nivel de la fístula no hay pérdidas urinarias. Otra forma de presentación clínica, en relación con la menstruación, es la hematuria cíclica (menuria). Esta forma clínica infrecuente, cuando se asocia a amenorrea, infertilidad y continencia urinaria, se denomina síndrome de Youssef.

La afectación de la fertilidad posterior se ve raramente comprometida una vez resuelta la fístula ${ }^{9}$. 
Una historia clínica sugestiva es un primer paso para sospechar la presencia de una comunicación vésicouterina, pudiéndose demostrar de forma indirecta mediante un test de incontinencia con azul de metileno o índigo carmín.

Pruebas de imagen con contraste, como la cistografía o la histerografía, son válidas para localizar la extensión y situación del trayecto fistuloso, aunque pueden presentar falsos negativos si la presión de la inyección del contraste no es la adecuada para forzar la apertura de la fístula. En muchas ocasiones la uretrocistoscopia nos identifica claramente la localización y su relación con los orificios ureterales. Por otro lado, en todo estudio de fístulas urogenitales es conveniente la realización de urografía intravenosa para valorar la integridad del aparato urinario superior.

Estas fístulas no suelen cerrar espontáneamente, y casi siempre, la reparación quirúrgica se impone mediante exéresis del trayecto fistuloso, cierre de la vejiga y del útero por planos ${ }^{10}$, siendo la vía más frecuentemente utilizada la abdominal transperitoneal de O'connor.

Si bien la cirugía es la técnica habitual, el tratamiento conservador mediante sondaje permanente durante dos o tres semanas puede ser resolutivo en pequeñas fístulas.

La fulguración mediante cistoscopia de los bordes de la fístula es otra opción antes de tomar una actitud quirúrgica ${ }^{11}$.

Jozwik $^{12}$ consigue el cierre espontáneo en ocho casos de nueve fístulas, mediante inducción de amenorrea con tratamiento hormonal.

En definitiva, y dado que la causa principal es la cesárea, la mejor prevención consiste en realizar una correcta indicación de la misma, así como practicar una cuidadosa técnica quirúrgica.

\section{REFERENCIAS}

1. LENKOUSKY $Z$, PODE D, SHAPIRO A, CAINE M.: Vesicouterine fistula: a rare complication of caesarean section. $J$ Urol 1988; 139: 123-125.

2. JÓZWIK M, JÓZWIK M, LOTOCKI W.: Actual incidence and cause of vesico-uterine fistula. $\mathrm{Br} J$ Urol 1998; 81: 341-342.

3. TÁNCER ML.: Vesicouterine fistula - a review. Obstet Gynecol Surv 1986 dec; 41 (12): 743-753.

4. YIP SK, LEUNG TY.: Vesicouterine fistula: an updated review. Int Urogynecol $J$ Pelvic Floor Dysfunct 1998; 9 (5): 252-256.

5. KÄSER, HIRSCH, IKLÉ.: Cirugía de las fístulas del tracto urinario inferior. En Atlas de Cirugía Ginecológica. Editorial Marban: 585, 5o edición.

6. MORENO R, ALOMAR A, USANDIZAGA A, CARTAÑÁ J.: Fístula vésicouterina: análisis de seis casos de una rara entidad: progresos de Obstetricia y Ginecología. Agosto 2000; 43 (8): 410-415.

7. JOZWIK M, JOZWIK M, LOTOCKI W.: Vesicouterine fistula - an analysis of 24 cases from Poland. Int $J$ Gynaecol Obstet 1997 may; 57 (2): 169-172.

8. SOLER SOLER JL, NAVARRO REPISO JA, HIDALGO DOMÍNGUEZ R et al.: Fístula vésico-uterina: aportación de dos nuevos casos. Actas Urol Esp 1998 feb.: 146-149.

9. EL MOUSSAOUI A, ABOUTAIEB R, BENNANI S, ELMRINI M, MEZIANE F, BENJELLOUN S.: Vesico-uterine fistulas. Analisis of 19 cases. J Urol 1994; 100 (3): 143-146.

10. GÓMEZ VEGAS A, BLÁZQUEZ IZQUIERDO J, SILMI MOYANO A.: Aspectos generales, clínica y diagnóstico de las fístulas ginecológicas. Bases terapéuticas. En Clínicas Urológicas de la Complutense. Fístulas Aparato Génitourinario: 313-319.

11. FERNÁNDEZ RODRÍGUEZ A, GÓMEZ BALAGUER M, MONTANANA V, SANTOLAYA GARCÍA JI, POLO PERIS A, CANTO FAUBEL E.: Fístula vésicouterina. Tratamiento conservador. Arch Esp Urol 1991; en-feb 44 (1): 80-81.

12. JOZWIK M , JOZWIK M.: Spontaneous closure of vesicouterine fistula. Account for effective hormonal treatment. Urol Int 1999; 62 (3): 183-187.

Dr. N. Chesa Ponce

Servicio de Urología

Hospital Universitario Insular de Gran Canaria

Avda. Maritima del Sur, s/n

35016 Las Palmas de Gran Canaria

(Trabajo recibido el 10 de junio 2002) 